Diseases

Editorial Manager(tm) for European Journal of Clinical Microbiology \& Infectious

Manuscript Draft

Manuscript Number: EJCMID-D-09-00369R1

Title: Management of post-splenectomy patients in the Netherlands.

Article Type: Article

Keywords: splenectomy; immunizations; sepsis; management

Corresponding Author: Ms. Jolanda Lammers, M.D.

Corresponding Author's Institution: Academic Medical Center

First Author: Jolanda Lammers, MD

Order of Authors: Jolanda Lammers, MD; D Veninga; M J Lombarts; J B Hoekstra; P Speelman

Abstract: Purpose.

After splenectomy, patients are at increased risk of sepsis with considerable mortality. The risk of sepsis can be reduced by immunizing these patients and by prescribing antibiotic prophylaxis. The purpose of our study was to determine compliance with the international standards for the management of splenectomised patients in the Netherlands, by investigating (i) vaccination rates, (ii) prescription of antibiotics and (iii) information in discharge letters.

Methods.

A retrospective review of medical records and discharge correspondence of 609 splenectomy patients from 1997 to 2008 was performed. Data were collected from 28 hospitals. Adherence to vaccination guidelines and the prescription of antibiotics were assessed.

Results.

$85.4 \%$ of post-splenectomy patients received pneumococcal vaccination, $39.4 \%$ received $\mathrm{H}$. Influenzae type $\mathrm{B}$ and $32.3 \%$ received meningococcal group $\mathrm{C}$ vaccination. $12.4 \%$ of patients were discharged on prophylactic antibiotics. In less than $25 \%$ of cases adequate recommendations regarding postsplenectomy management were given to the general practitioner.

Conclusions.

In the Netherlands compliance with recommendations for management of patients after splenectomy is insufficient. Fifteen percent does not receive vaccination against pneumococci and the majority of patients does not receive antibiotic prophylaxis. The development and implementation of a national guideline for splenectomised patients is urgently required.

Response to Reviewers: Response to Reviewers:

1. The Results section is clear but perhaps the categories of splenectomy could more easily be divided into Emergency and Elective, with further sub-division of Emergency into non-iatrogenic and iatrogenic and Elective into planned pre-operative and planned peri-operative.

Answer:

At present, in the manuscript the following 4 categories are used: 
A. acute/emergency splenectomy

B. elective/planned splenectomy

C. iatrogenic/inadvertent splenectomy

D. durante operatione.

The suggestion of reviewer \#1 is to change the categories into:

A. emergency splenectomy, further divided into iatrogenic vs. non-iatrogenic

B. elective splenectomy, further divided into planned pre-operatively and planned peri-operatively.

We have considered this division during our data-analysis as well, but concluded the definitions would than not be appropriate for the following reasons:

1. In our definition, not all iatrogenic splenectomies are emergency splenectomies: in our acute/emergency category only those surgeries were included where there was spontaneous bleeding of the spleen with shock, rupture of an aneurysm of the splenic artery, or trauma to the spleen caused for example by high-energetic trauma. Splenectomies due to bleeding caused by the surgeon when operating for another indication were not included here. In our opinion, these are different categories, for example differing in setting (multiple trauma/acute operation versus scheduled bowel-surgery) and operating staff (trauma surgeons versus surgeons specialized in stomach/esophagus surgery).

2. The category of elective splenectomy cannot be divided into "planned peri-operatively", since we are investigating timing of vaccination. When a splenectomy is planned, ideally the vaccination should be given 2 weeks prior to the surgery. If it is decided during surgery to remove the spleen, preoperative vaccination has not been performed.

2. The authors have not indicated whether there was any change in practice over time Answer:

These data are readily available in our database. We decided to omit these data considering length of the manuscript, but we have added them as suggested by reviewer \#1. See table 5.

3. nor were there any differences in management by institution (eg. Teaching versus nonTeaching).

Answer:

We acknowledge the lack of institutional data in this manuscript, as suggested by reviewer \#1. However, considering the length of the manuscript, as well as relevance to the EJCMID, we have decided to accumulate these management data in a second manuscript (including data regarding hospital teaching status, but also the influence of the presence of hospital guideline or hospital protocol on hospital performance in the prevention of infections, as well as the influence of the number of surgeons in the hospital staff on performance). This second manuscript is currently under revision at the Journal of Hospital Medicine.

4. Data on Emergency versus Elective procedures is available for vaccination but not antibiotic prescribing. Although one would predict differences in these areas confirmation of the influence of these measurables on out-come would be of some interest. 
Answer:

Again, we acknowledge the lack of these data in the manuscript, as suggested by reviewer \#1. Considering the low antibiotic prescription rates, we considered these numbers not to be as relevant as for vaccination status. However, these data can and have been added to the manuscript. See table 4 . 


\section{1 \\ Management of post-splenectomy patients in the Netherlands}

Corresponding author:

A.J.J. Lammers, Department of Infectious Diseases, Tropical Medicine and AIDS, G2-

105, Academic Medical Center, Meibergdreef 9, 1105 AZ, Amsterdam, Netherlands.

Tel.(+31)-20-566 7062 / 64380, fax: (+31)-20-6972286.

Email: a.j.lammers@amc.uva.nl

Affiliations and addresses of other authors:

D. Veninga, Department of Infectious Diseases, Tropical Medicine and AIDS, F4-217, Academic Medical Center, Meibergdreef 9, 1105 AZ, Amsterdam, Netherlands.

M.J.M.H. Lombarts, PhD, Department of Quality and Process Innovation, J2-211, Academic Medical Center, Meibergdreef 9, 1105 AZ, Amsterdam, Netherlands.

J.B.L. Hoekstra, professor, Department of Internal Medicine, F4-217, Academic Medical Center, Meibergdreef 9, 1105 AZ Amsterdam, Netherlands

P. Speelman, professor, Department of Infectious Diseases, Tropical Medicine and AIDS, Academic Medical Center, F4-217, Meibergdreef 9, 1105 AZ Amsterdam, Netherlands. 


\section{Introduction}

The spleen has an important function in the host protection against invading micro-organisms [1]. Splenic marginal zone macrophages filter and phagocytose bacteria and other blood-borne pathogens from the blood that flows through the splenic red pulp [2]. After splenectomy there is a diminished clearance of poorly opsonized bacterial antigens, an impaired primary humoral response to antigenic challenge and loss of the vagally mediated anti-inflammatory cholinergic response $[3,4]$.

The asplenic state thus results in a serious impairment of clearance of mainly encapsulated bacteria, which can lead to a life-threatening infection known as post-splenectomy sepsis, PSS $[5,6]$. The encapsulated bacterium Streptococcus pneumoniae is the causative pathogen in $80 \%$ of cases of PSS $[7,8]$. Although often preceded by nonspecific symptoms, PSS deteriorates in hours rather than days to a severe septic state that is associated with a high mortality of 50-70\% $[7,9,10]$. The incidence of sepsis in post-splenectomy patients is estimated to be 600 times greater than in the general population [11]. The incidence of invasive infection among children and adults after splenectomy is similar, $3.3 \%$ and $3.2 \%$ respectively [12].

Several relevant organizations developed recommendations to prevent this syndrome [13].

Evidence supporting these recommendations is mostly based on clinical studies (retrospective studies, series of case-reports) and expert opinion. Unfortunately, data from randomized controlled intervention trials are not available. In the Netherlands, a guideline for the management of post-splenectomy patients has not yet been developed.

In 1996, the British Committee for Standards in Haematology published Guidelines for the prevention and treatment of infection in patients with an absent or dysfunctional spleen [14], that are considered to reflect best-practice. These recommendations were updated in 2002 [15], and consist of the following key elements: 
1. All splenectomised patients should receive pneumococcal immunization and revaccination. If not previously immunized they should also receive Haemophilus influenzae type B and meningococcal $\mathrm{C}$ vaccine. Yearly influenza immunization is recommended.

2. Lifelong prophylactic antibiotics are recommended, or at least the first two years after splenectomy. In case of suspected or proven infection patients should be given systemic antibiotics and be admitted to a hospital.

3. All patients should be educated about the risks of infection and the risk associated with traveling (such as becoming infected by Plasmodium falciparum) and unusual infections (i.e. dog bites). Patient records should be labeled to indicate the risk.

It has become clear from recent literature that post-splenectomy patients are not all being managed according to best practice $[5,8,9,16-21]$

The goal of our study was to determine the management of post-splenectomy patients for the first time in the Netherlands. We therefore assessed compliance with the British best practice standards by investigating (i) vaccination rates, (ii) whether or not antibiotics were prescribed and (iii) if the hospital provided the general practitioner with relevant information regarding the period after the operation. 


\section{Methods}

\section{Hospital and patient inclusion}

Splenectomised patients were included retrospectively from a representative sample of Dutch hospitals. First, $30 \%$ of all Dutch hospitals were identified through a blind drawing, including academic, teaching and non teaching hospitals. Subsequently, patients were identified using the Dutch Pathology Registry, since spleens are routinely sent to the pathology department after removal. In the Registry, a search query *milt* (spleen) was performed, after which all splenectomies performed from 1997 to 2008 were selected and non-relevant hits such as partial splenectomies or spleen biopsies were removed. Inclusion started with the most recent splenectomy, subsequently going back in time in a consecutive matter. After hospitals and patients were identified, the medical records and all discharge correspondence were assessed on site. In case the medical file or discharge correspondence was not available, the patient was excluded. To investigate discharge correspondence, we included discharge letters as well as all other correspondence up to at least 1 year after splenectomy, for example from follow-up outpatient visits.

\section{Used definitions}

After inclusion, patients were divided into 4 categories of splenectomy: 'acute' in case of emergency splenectomies, 'elective' in case of planned splenectomies, 'iatrogenic' in case of inadvertent splenectomies and 'durante operatione' in case it was decided during the operation to perform a non-inadvertent splenectomy.

Vaccination was considered to be correct if 3 immunizations were given: pneumococcal, $H$. influenzae $\mathrm{B}$ and meningococcal group $\mathrm{C}$ vaccination. Pneumococcal vaccination was defined as immunization with either the 23-valent pneumococcal polysaccharides vaccine (PPV-23, Pneumovax), the 7-valent pneumococcal conjugate vaccine (PCV-7, Prevenar), or both. The 
British guidelines recommend that pneumococcal vaccination is given either 2 weeks prior to or 2 weeks after splenectomy. In this study, timing was considered correct if given either 1-3 weeks prior to surgery in case of elective splenectomies, or 1-3 weeks after the operation.

Prescription of antibiotics was considered to be correct if antibiotic prophylaxis was given for the first 2 years after splenectomy, in combination with a prescription for on-demand (broad spectrum) antibiotics to be taken in case of suspected infection.

\section{Data collection method}

All data were collected by the same two investigators (DV, JL), using a standardized survey form. Individual data included patient demographics, indication for splenectomy, documentation of vaccine administration, timing of vaccination in relation to splenectomy and documentation of post-splenectomy infections.

Furthermore, discharge correspondence was checked for mentioning of each of the following: performed splenectomy, vaccination status, the need for revaccination, prescribed prophylactic antibiotics, the need of urgent use of antibiotics in case of suspected infection and the advice for annual flu-vaccination.

\section{Statistics}

Analysis of data was performed in SPSS 16.0. Confidence intervals were computed using CIA (Confidence Interval Analysis) version 1.0, copy 5/0618 (Gardner, British Medical Journal). 


\section{Results}

In the period 1997 to 2008 a total number of 609 splenectomy cases from 28 hospitals were identified. 73 cases were excluded: 50 hospital records were not available and 23 patients could not be identified (transferred to another hospital, patient not known, no permission from patient or hospital to access the medical file). 536 patients were included (see table 1), with a mean duration of follow-up of 4.22 years. In total, 19 children under the age of 18 were included.

Of the 185 splenectomies of iatrogenic cause, injury to the spleen was most likely to occur in stomach/esophagus operations (32.4\%), in operations of the colon descendens (18.4\%) and rectum-sigmoid (11.4\%) and operations of the left kidney (14.6\%).

\section{Vaccination}

To study vaccination rates, we omitted patients that died within 2 weeks after the operation $(\mathrm{n}=36)$. Data regarding actual vaccination was missing for 1 pneumococcal vaccination and for 2 cases of the other immunizations. Overall, 426 of 499 patients $(85.4 \%)$ received pneumococcal vaccination. 196 (39.4\%) patients received $H$. influenzae type B vaccination and 161 (32.3\%) received the meningococcal group $\mathrm{C}$ vaccine. $30.5 \%$ of patients in our study received all 3 recommended vaccines.

Pneumococcal-vaccination rates in relation to type of operation are shown in table 2. Rates were highest for patients that underwent an acute or elective splenectomy. If the operation was performed for another indication than a spleen extirpation, such as in iatrogenic or duranteoperatione splenectomies, these rates were significantly lower (CI-95\% $0.158-0.307$ for elective vs. iatrogenic splenectomy).

Of the 184 patients that underwent an elective splenectomy and received pneumococcal vaccination, 144 patients received their immunization preoperatively (78.3\%) and 31 postoperatively (16.7\%). For 9 patients vaccination timing was not documented. 
Of all preoperatively vaccinated patients, in 12 cases $(8.3 \%)$ the immunization was given 1-3 weeks prior to surgery, another 12 cases $(8.3 \%)$ were vaccinated in the week before surgery, 13 (9\%) were vaccinated 3 weeks to 6 months before surgery, and of 108 patients (74.5.\%) the exact timing of vaccination was not documented.

For immunizations given postoperatively, in $169(62.4 \%)$ the timing of vaccination was not documented. Of the remaining 107, 30 vaccinations (28\%) were given 1-3 weeks after surgery, 3 vaccinations were given on the day of surgery, 34 within a week after surgery and 40 vaccinations were given 1 - 33 months after splenectomy.

\section{Antibiotics}

The majority of patients was discharged from the hospital without a prescription for antibiotics (see table 3). The duration of antibiotic prophylaxis is shown in figure 1. Of the 77 patients receiving prophylaxis, 24 patients $(31.2 \%)$ received antibiotics for 2 years, 1 patient received a life-long prescription because of an underlying immunodeficiency, 2 children received prophylaxis until they reached 16 years of age and in 11 cases $(14.3 \%)$ the duration of prophylaxis was not-documented. In 6 cases (7.8\%) antibiotics were given "until vaccination" (3 cases length unknown, 3 cases $<1$ month). Table 4 shows prescription rates of antibiotics in relation to type of operation.

\section{Change in time}

In table 5, the change in vaccination rates (5A) and prescription of antibiotics (5B) over time is shown. We have decided to compare the period before and after 2002, since at that time the update of the British guideline was published. After 2002, there is a significant increase in vaccination with $\mathrm{H}$. influenzae and meningococci $\mathrm{C}$ and a significant decrease in patients not receiving any antibiotics, due to more prescribed "ondemand" antibiotics. 


\section{Information in discharge correspondence}

The specific information mentioned in discharge correspondence to the general practitioner (GP) is summarized in table 6. Splenectomy and given immunizations were mentioned in the majority of patients whereas other recommended advises were found in a minority of cases.

\section{Infectious complications}

The number of patients that developed a documented infection after splenectomy was 295 (55\%), during a mean period of follow-up of the cohort of 4.22 years (range $0-12$ ). Infection was defined as any infectious complication found in hospital medical files and medical correspondence for each patient. Of these 295 patients, 278 (94.2\%) developed their first infection within 2 years after splenectomy.

Infectious complications were distributed over the splenectomy indications as follows: 121 patients (41\%) developed an infection after a splenectomy of iatrogenic cause, 90 patients (31\%) after elective surgery, 60 patients (20\%) after acute splenectomy and $24(8 \%)$ if it was decided durante operatione to perform a splenectomy.

Since asplenic hosts are particularly susceptible for blood borne infections, we investigated the incidence of bacteraemias in these patients. We found that $12.5 \%$ of patients developed a documented bacteraemic episode after splenectomy (i.e. 67 of 536 patients). A total of 96 bacteraemias occurred in these 67 patients. Pathogens responsible for these bacteraemias were identified in 61 episodes and are shown in table 7. No meningococcal bacteraemia was documented. Other infections included pneumonias (155, of which 17 were proven positive for either S. pneumoniae or $H$. influenzae), urinary tract infections (86), fever of unknown origin (78) and miscellaneous causes (149) such as abdominal abscesses, wound infection and diarrhea. Patient-characteristics of documented pneumococcal and $H$. influenzae bacteraemias after splenectomy are shown in table 8 . 


\section{Discussion}

Although vaccination results in the Netherlands are among the highest reported in literature, it is still of concern that $14.6 \%$ of post-splenectomy patients was not vaccinated against pneumococci, $60.6 \%$ of splenectomised patients did not receive vaccination against $H$. influenzae, and $67.7 \%$ did not receive meningococcal vaccination.

We found only $12.4 \%$ of included patients to have received a prescription for prophylactic antibiotic therapy, of which $40.9 \%$ was of correct duration. The role and efficacy of prophylactic antibiotics after splenectomy remains unclear and their use is not generally recommended [6]. However, in the absence of evidence against it, the British Guidelines recommend to offer lifelong prophylaxis with oral phenoxymethylpenicillin. If there is hesitation to prescribe lifelong antibiotics, it is recommended to cover at least the first two years after splenectomy, as well as children aged up to 16 . In most hospitals included in this survey, antibiotic prophylaxis is not recommended and compliance even for these first two years is estimated to be low.

Reviewing discharge correspondence, it is encouraging to note that in the majority of cases splenectomy status $(97.7 \%)$ was documented. It is however unfortunate that of all immunized patients in $20 \%$ the vaccination was not mentioned to the general practitioner and that other recommendations were given only in a minority of cases. Primary care doctors, who are also responsible for keeping patients fully immunized, should be informed about necessary vaccinations, the importance of antibiotics and the measurements that should be taken in case of a potential PSS.

\section{Infections after splenectomy}

Although very relevant, our study was not designed to describe the effectiveness of vaccination or antibiotics on reducing infections after splenectomy. In a recent review, it is described that non- 
vaccinated patients have a higher incidence of infection then vaccinated patients [13]. In some studies this difference was not significant, however overall there does appear to be both a statistical and a clinically relevant difference.

More than half of the patients in our cohort developed a documented infection after surgery. Most infections occurred after an iatrogenic splenectomy. It is however likely for patients to have a higher risk of infection after major abdominal surgery then after a splenectomy only. The incidence of bacteraemia in splenectomised patients in this study was $12.5 \%$ during an average follow up period of 4.22 years after splenectomy. There is evidence that besides the known susceptibility of asplenic hosts for encapsulated bacteria, other organisms such as Gram-negative bacteria and Capnocytophaga canimorsus cause infections and carry considerable morbidity $[5,10]$. In a study by Holdsworth and colleagues, it is demonstrated that of 349 postsplenectomy infections E. coli and Pseudomonas, although with low incidence, carried the highest mortality [7]. Although the causative microorganisms in our study were not always identified, we demonstrate that indeed not only encapsulated pathogens play a role in postsplenectomy bacteraemia, but that asplenic hosts are also at risk for staphylococci, enterococci, E. coli, and other pathogens. For the bacteraemias caused by coagulase-negative staphylococci however, it remains unclear whether these pathogens have caused a clinical relevant infection. $N$. meningitidis has been cited as the third most common cause for PSS [7]. Even though meningococcal vaccination rates were low, we have found no bacteraemic episodes based on meningococcal infection. In our study only one case of meningitis was documented, which was due to S. aureus.

Despite voluminous literature on post-splenectomy infection, estimates of bacteraemic risk vary and precise figures remain unclear. This is in part due to variation in definitions of PSS, variation in duration of follow-up, lacking documentation and heterogeneity and small sample sizes of study populations. 


\section{Limitations}

There are some limitations to our investigation. First, there are several aspects of our study that are likely to bias the outcome: the large variation in duration of follow-up (range from several months to 12 years), patients being lost to follow-up due to transfer to another hospital of care, (severe) infections or death under supervision of the GP thus not identified in our study, all leading to an underestimation of the true incidence of infection and death. This possible underestimation would result in unreliable conclusions on the effects of preventive measures in infection and death after splenectomy in our cohort.

Furthermore, we have not contacted patients to check for their knowledge about the need for (re)vaccination, antibiotics and precautions to be taken when travelling. Informing patients is considered to be a major factor in improving management [15]. Another consequence is that although booster vaccinations, prophylactic or on-demand antibiotics may have been recommended, we are not informed about patients' and GP's compliance. This may cause an overestimation of antibiotic use and revaccination rate.

Finally, although we demonstrate vaccination rates, it remains unclear whether these vaccinations were effective, since 5-10\% of healthy adults fail to produce antibody to most or all polysaccharides in PPV23 [22]. Ideally, patients should be tested with titer-analysis for adequate antibody levels. Although there are no large scale, randomized controlled intervention trials that have tested the efficacy of bacterial vaccines in preventing PSS, data from studies that reported antibody levels measured before and after vaccination, suggest that the risk of infection in vaccinated patients is higher in those with a poor immunological response [23].

\section{Conclusion}

We have investigated the management of post-splenectomy patients in the Netherlands for the first time. Although the pneumococcal vaccination rate after splenectomy is among the highest 
reported in literature, hospitals could do much better at providing general practitioners with recommendations for post-splenectomy management.

Future research will have to investigate the reason for this non-compliance with international guidelines. It is important that all caregivers aim at $100 \%$ vaccination rates and all patients being discharged with antibiotics and clear instructions on how and when to use them. Therefore, we emphasize the need for a Dutch guideline for this group of patients. 


\section{Acknowledgements}

This study was supported by ZonMw.

We would like to thank all participating hospitals for their cooperation.

There are no potential conflicts of interest for any of the authors. 


\section{References}

\section{Morris DH, Bullock FD (1919) THE IMPORTANCE OF THE SPLEEN IN RESISTANCE TO INFECTION. Ann.Surg. 70:513-521.}

2. Mebius RE, Kraal G (2005) Structure and function of the spleen. Nat.Rev.Immunol. 5:606-616.

3. Hosea SW, Brown EJ, Hamburger MI et al (1981) Opsonic requirements for intravascular clearance after splenectomy. N.Engl.J.Med. 304:245-250.

4. Huston JM, Ochani M, Rosas-Ballina M et al (2006) Splenectomy inactivates the cholinergic antiinflammatory pathway during lethal endotoxemia and polymicrobial sepsis. J.Exp.Med. 203:1623-1628.

5. Deodhar M, Kakkar N (2004) An audit of splenectomies in a teaching hospital in North India. Are postsplenectomy guidelines being complied with? J.Clin.Pathol. 57:407410.

6. Newland A, Provan D, Myint S (2005) Preventing severe infection after splenectomy. Bmj 331:417-8.

7. Holdsworth RJ, Irving AD, Cuschieri A (1991) Postsplenectomy sepsis and its mortality rate: actual versus perceived risks. Br J Surg 78:1031-8.

8. Waghorn DJ (2001) Overwhelming infection in asplenic patients: current best practice preventive measures are not being followed. J Clin Pathol 54:214-8.

9. Brigden ML, Pattullo A, Brown G (2000) Pneumococcal vaccine administration associated with splenectomy: the need for improved education, documentation, and the use of a practical checklist. Am J Hematol 65:25-9.

10. Styrt B (1990) Infection associated with asplenia: risks, mechanisms, and prevention. Am.J.Med. 88:33N-42N.

11. Singer DB (1973) Postsplenectomy sepsis. Perspect.Pediatr.Pathol. 1:285-311.:285311.

12. Bisharat N, Omari H, Lavi I et al (2001) Risk of infection and death among postsplenectomy patients. J Infect 43:182-6.

13. Mourtzoukou EG, Pappas G, Peppas G et al (2008) Vaccination of asplenic or hyposplenic adults. Br.J.Surg. 95:273-280.

14. (1996) Guidelines for the prevention and treatment of infection in patients with an absent or dysfunctional spleen. Working Party of the British Committee for Standards in Haematology Clinical Haematology Task Force. BMJ. 312:430-434. 
15. Davies JM, Barnes R, Milligan D (2002) Update of guidelines for the prevention and treatment of infection in patients with an absent or dysfunctional spleen. Clin Med 2:440-3.

16. Fuentes-Ferrer ME, Cano-Escudero S, Mato-Chain G et al (2008) [Vaccination coverage against Streptococcus pneumoniae in splenectomized patients in a fourthlevel hospital (1999-2004)]. Enferm.Infecc.Microbiol.Clin. 26:194-198.

17. Hasse B, Moll C, Oehy K et al (2005) Anti-infectious prophylaxis after splenectomy: current practice in an eastern region of Switzerland. Swiss.Med.Wkly. 135:291-296.

18. Kyaw MH, Holmes EM, Chalmers J et al (2002) A survey of vaccine coverage and antibiotic prophylaxis in splenectomised patients in Scotland. J.Clin.Pathol. 55:472474.

19. O'Donnell J, McGreal G, Daly P et al (2004) Management of patients undergoing splenectomy in an Irish teaching hospital: impact of guidelines. Ir.J.Med.Sci. 173:136140.

20. Pickering J, Campbell H (2000) An audit of the vaccination and antibiotic prophylaxis practices amongst patients splenectomised in Lothian. Health Bull.(Edinb.). 58:390-395.

21. Ramachandra J, Bond A, Ranaboldo C et al (2003) An audit of post-splenectomy prophylaxis--are we following the guidelines? Ann R Coll Surg Engl 85:252-5.

22. Musher DM, Ceasar H, Kojic EM et al (2005) Administration of protein-conjugate pneumococcal vaccine to patients who have invasive disease after splenectomy despite their having received 23 -valent pneumococcal polysaccharide vaccine. J.Infect.Dis. 191:1063-1067.

23. Cherif H, Landgren O, Konradsen HB et al (2006) Poor antibody response to pneumococcal polysaccharide vaccination suggests increased susceptibility to pneumococcal infection in splenectomized patients with hematological diseases. Vaccine. 24:75-81. 


\section{Tables}

Table 1. Demographics.

\begin{tabular}{ll}
\hline Patients [n] & $\mathbf{5 3 6}$ \\
\hline Patients per hospital type [n (hospitals)] & \\
Academic & $20(2)$ \\
Teaching & $209(11)$ \\
Non-teaching & \\
Gender [n (\%)] & $276(51,5)$ \\
Male & $260(48,5)$ \\
Female & $64(5-91)$ \\
Median age at operation [years (range)] & \\
Indication for splenectomy [n (\%)] & $115(21,4)$ \\
Acute & $196(36,5)$ \\
Elective & \\
Iatrogenic lesion & \\
Durante operatione & \\
\hline
\end{tabular}


Table 2. Vaccination results for pneumococcal vaccines given to patients according to operation indication.

\begin{tabular}{lll}
\hline Indication for splenectomy & Total & Vaccinated; $\mathrm{n}(\%)$ \\
\hline Acute & 104 & $96(92.3)$ \\
Elective & 193 & $184(95.3)$ \\
Iatrogenic & 165 & $119(72.1)$ \\
Durante operatione & 37 & $27 \quad(73)$ \\
\hline Total $^{\text {a }}$ & 499 & $426(85.4)$ \\
\hline
\end{tabular}

${ }^{\mathrm{a}}$ Total number of patients only includes patients that have survived the first two weeks after splenectomy. 
Table 3. Prescribed antibiotics after splenectomy.

\begin{tabular}{lll}
\hline Antibiotics & Number of patients & $\%$ \\
\hline Prophylactic $^{\mathrm{a}}$ & $62 / 500$ & 12.4 \\
On-demand & $41 / 500$ & 8.2 \\
Both & $15 / 500$ & 3.0 \\
N.A. ${ }^{\text {b }}$ & $31 / 500$ & 6.2 \\
No antibiotics & $351 / 500$ & 70.2 \\
\hline
\end{tabular}

\footnotetext{
${ }^{\mathrm{a}}$ Defined as a prescription for prophylactic antibiotics given to the patient at time of discharge.

${ }^{b}$ N.A. (not applicable) refers to patients that died in the hospital before discharge, but at least 2 weeks after splenectomy.
} 
Table 4. Prescription rates of antibiotics according to operation indication.

\begin{tabular}{lcccc}
\hline Antibiotics (\%) & prophylactic & on-demand & both & none \\
\hline Indication for splenectomy & & 10.1 & 2 & 68.7 \\
Acute & 18.3 & 11 & 4 & 73.3 \\
Elective & 11.4 & 5.6 & 2.8 & 81.1 \\
Iatrogenic & 9.1 & 5.6 & 2.8 & 75 \\
Durante operatione & 15.8 & & & \\
\hline
\end{tabular}


Table 5. Changes in vaccination rates and antibiotic prescription over time.

5A. Vaccination rates before and after 2002.

\begin{tabular}{llll}
\hline Vaccination (\%) & $\mathbf{1 9 9 7 - 2 0 0 1}$ & $\mathbf{2 0 0 2 - 2 0 0 8}$ & CI 95\% \\
\hline pneumococcal & 88.2 & 84.8 & -0.0422 to 0.111 \\
$\begin{array}{l}\text { H. influenzae } \\
\text { meningococcal C }\end{array}$ & 15.3 & 44.3 & -0.380 to -0.200 \\
\hline CI $=$ confidence interval & 3.5 & 38.3 & -0.408 to -0.286 \\
\hline
\end{tabular}

5B. Prescription of antibiotics before and after 2002.

\begin{tabular}{llll}
\hline Antibiotics (\%) & $\mathbf{1 9 9 7 - 2 0 0 1}$ & $\mathbf{2 0 0 2 - 2 0 0 8}$ & CI 95\% \\
\hline prophylactic & 10.6 & 12.8 & -0.0947 to 0.0510 \\
on-demand & 3.7 & 9.8 & -0.112 to -0.0113 \\
both & 0 & 3.9 & 0.0195 to 0.0580 \\
none & 85.4 & 72.6 & 0.0391 to 0.216 \\
\hline CI = confidence interval & & &
\end{tabular}


Table 6. Information in discharge correspondence to general practitioner

\begin{tabular}{lcc}
\hline All correspondence & Number of patients & $\%$ \\
\hline Mention splenectomy & $458 / 469$ & 97.7 \\
Mention of vaccination ${ }^{\text {a }}$ & $343 / 426$ & 80.5 \\
Advice revaccination & $110 / 469$ & 23.5 \\
Advice annual flu-vaccination & $59 / 469$ & 12.6 \\
Advice on-demand antibiotics & $100 / 469$ & 21.3 \\
\hline
\end{tabular}

Numbers are minus 36 patients that died within 2 weeks after splenectomy and minus 31 patients that died before being discharged from the hospital.

a only of those cases in which pneumococcal vaccination was given 
Table 7. Distribution of pathogens in post-splenectomy bacteraemia.

\begin{tabular}{|c|c|}
\hline Pathogen & Number of positive bloodcultures \\
\hline S. pneumoniae & 6 \\
\hline H. influenzae & 1 \\
\hline S. aureus & 6 \\
\hline $\mathrm{CNS}^{\mathrm{a}}$ & 16 \\
\hline Enterococci $^{b}$ & 9 \\
\hline E. coli & 7 \\
\hline Pseudomonas & 2 \\
\hline Klebsiella $^{\mathrm{c}}$ & 3 \\
\hline Proteus mirabilis & 2 \\
\hline ESBL & 1 \\
\hline Miscellaneous $^{\mathrm{d}}$ & 8 \\
\hline \multicolumn{2}{|c|}{${ }^{\mathrm{a}}$ S. epiderdimis 3, MRSE 3, CNS 6, undefined 4} \\
\hline \multicolumn{2}{|c|}{${ }^{\mathrm{b}}$ E. faecium 5, E. faecalis 2 , sp. 2} \\
\hline \multicolumn{2}{|c|}{${ }^{\mathrm{c}} K$. pneumoniae $2, K$ oxytoca } \\
\hline
\end{tabular}


Table 8. Characteristics of patients with documented pneumococcal or $H$. influenzae bacteraemias after splenectomy.

\begin{tabular}{|l|l|l|l|l|l|}
\hline & age & reason for & vaccination & antibiotics & PSS \\
\hline Pt 1 & 64 & Iatrogenic & & & \\
& & $\begin{array}{l}\text { (during } \\
\text { colectomy) }\end{array}$ & 23-valent & none & - S. pneumoniae \\
after 5 months
\end{tabular}

ITP; idiopathic thrombocytopenic purpura, NHL; non-Hodgkin lymphoma, AIHA; autoimmune hemolytic anemia. 
Figure 1. Duration of prescribed prophylactic antibiotic therapy.

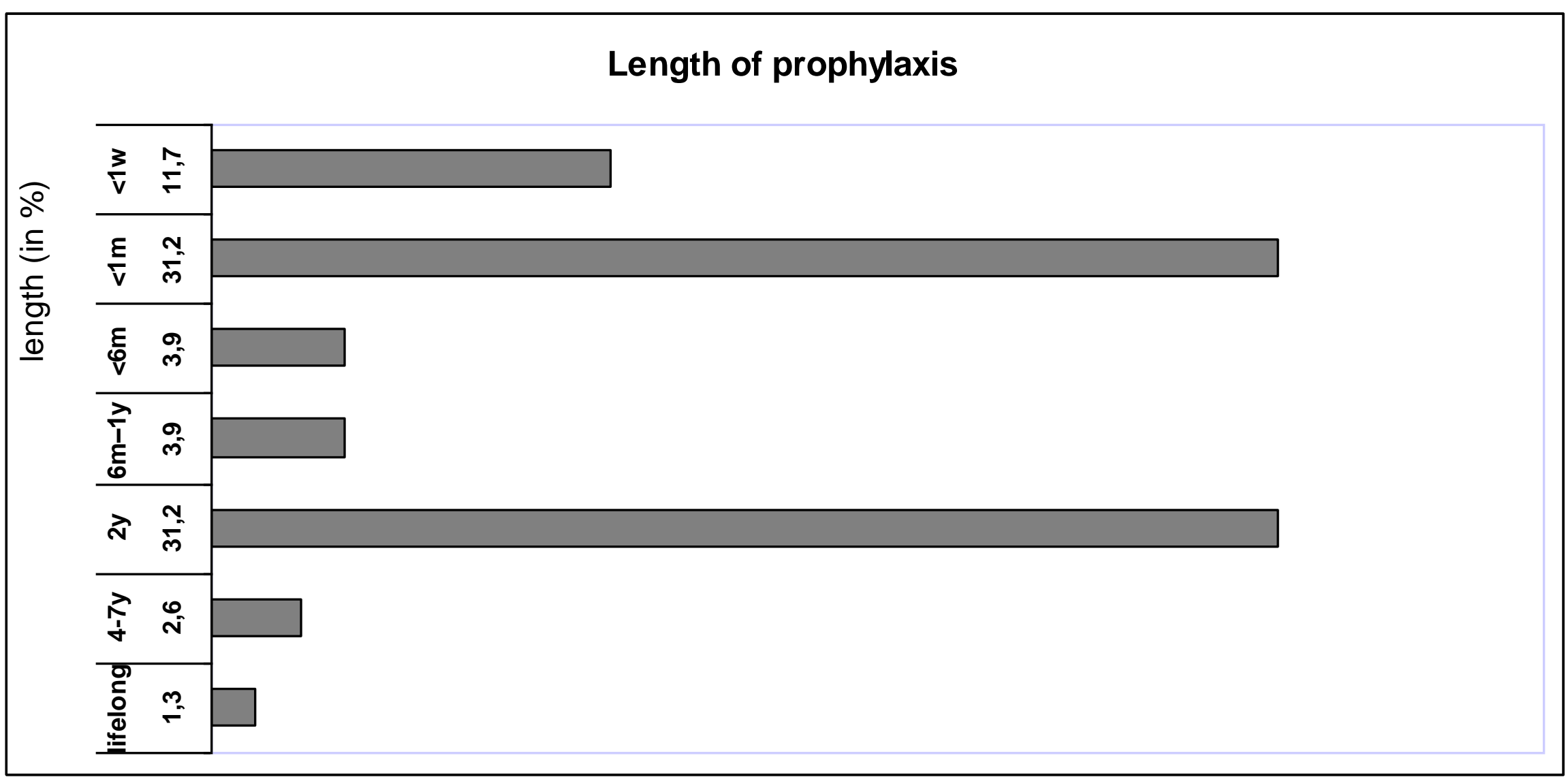

$\mathrm{w}=$ week, $\mathrm{m}=$ months, $\mathrm{y}=$ years. 